\title{
FITOSSANIDADE
}

\section{TOXICIDADE DE PRODUTOS FITOSSANITÁRIOS PARA ADULTOS DE ORIUS INSIDIOSUS (SAY) (HEMIPTERA: ANTHOCORIDAE) $\left({ }^{1}\right.$ )}

\author{
LUIZ CARLOS DIAS ROCHA $\left({ }^{2}\right)$; GERALDO ANDRADE CARVALHO $\left({ }^{3}\right)$; \\ ALEXANDRE PINHO MOURA $\left({ }^{4}\right)$; FABRÍCIA ZIMERMANN VILELA TORRES $\left({ }^{4}\right)$
}

\begin{abstract}
RESUMO
O objetivo do presente trabalho foi estudar a toxicidade de abamectin, acephate, azoxystrobin, benomyl, chlorfenapyr, imibenconazole, iprodione, metalaxyl + mancozeb e triforine para adultos de Orius insidiosus (Say). Os produtos foram utilizados nas concentrações indicadas pelos fabricantes e/ou que estão em fase de pesquisa para o controle de pragas e doenças na cultura do crisântemo, sendo aplicados em adultos por meio de torre de Potter. Os bioensaios foram realizados em laboratório, sob temperatura de $25 \pm 2{ }^{\circ} \mathrm{C}$, umidade relativa de $70 \pm 10 \%$ e fotofase de 12 horas. A toxicidade dos produtos para adultos foi determinada pela porcentagem do efeito total $(\mathrm{E} \%)$, levando-se em consideração a taxa de mortalidade e redução de oviposição, sendo, ainda, classificados segundo escala proposta por membros da IOBC. Abamectin, acephate e chlorfenapyr são tóxicos a adultos de O. insidiosus, provocando redução na sua sobrevivência. Fêmeas adultas de $O$. insidiosus tratadas com abamectin e acephate têm a viabilidade de seus ovos reduzida, enquanto a aplicação de abamectin e chlorfenapyr prolongam seu período de oviposição. Os fungicidas azoxystrobin, benomyl, imibenconazole, iprodione, metalaxyl + mancozeb e triforine têm baixa toxicidade ao $O$. insidiosus.
\end{abstract}

Palavras-chave: pesticidas, seletividade, cultivo protegido, controle biológico.

\begin{abstract}
PESTICIDE TOXICITY TO ADULTS OF ORIUS INSIDIOSUS (SAY) (HEMIPTERA: ANTHOCORIDAE)

The aim of this work was evaluate the toxicity of abamectin, acephate, azoxystrobin, benomyl, chlorfenapyr, imibenconazole, iprodione, metalaxyl + mancozeb and triforine on adults of Orius insidiosus (Say). These pesticides were used at the concentrations indicated by the manufacturers. All of them are in testing process for pest and diseases control on chrysanthemums crop, and were spraying on adults using a Potter's tower. The bioassays were carried out in laboratory, under controlled conditions at $25 \pm 2^{\circ} \mathrm{C}$,
\end{abstract}

( $\left.{ }^{1}\right)$ Recebido para publicação em 21 de março de 2005 e aceito em 3 de março de 2006.

$\left(^{2}\right)$ Doutorando em Entomologia. Departamento de Entomologia, Universidade Federal de Lavras (UFLA), Escola Agrotécnica Federal de Inconfidentes, Praça Tiradentes, 416, 37576-000 Inconfidentes (MG). E-mail: luizufla@gmail.com.

$\left({ }^{3}\right)$ Departamento de Entomologia, Universidade Federal de Lavras (UFLA), Caixa Postal 3037, 37200-000 Lavras (MG). E mail: gacarval@ufla.br.

$\left({ }^{4}\right)$ Doutorando em Entomologia, Universidade Federal de Lavras (UFLA), Lavras (MG). E-mail: alexandrepm@yahoo.com; fabriciazimermann@yahoo.com.br. Bolsistas CNPq. 
RH of $70 \pm 10 \%$ and 12 -h photophase. The toxicity of the pesticides to adults was determined by the total effect, considering the mortality rate and oviposition reduction. Toxicity effect was classified according to scale proposed by IOBC. Abamectin, acephate and chlorfenapyr were toxic to adults of O. insidiosus, and caused reduction in their survival. Females of $O$. insidiosus treated with abamectin and acephate caused reduction on eggs viability, whereas the application of abamectin and chlorfenapyr caused increase on pre-oviposition period. The fungicides azoxystrobin, benomyl, imibenconazole, iprodione, metalaxyl + mancozeb and triforine presented low toxicity to O. insidiosus.

Key words: pesticides, selectivity, protected crop, biological control.

\section{INTRODUÇÃO}

$\mathrm{Na}$ agricultura atual, exige-se cada vez mais, no mercado, a presença de produtos de alta qualidade e livres de resíduos de produtos fitossanitários. Aliados a esse fato, os riscos oferecidos ao aplicador de agroquímicos e a alta pressão de seleção no interior das casas de vegetação, proporcionando alto risco de surgimento de insetos-praga resistentes, são fatores que justificam o emprego de táticas de manejo de pragas menos agressivas ao meio ambiente. No manejo integrado de pragas (MIP), os produtos adotados para o combate de pragas devem causar o mínimo de impacto possível sobre os insetos considerados benéficos, para que possam colaborar com o produtor na eliminação de insetos-praga da cultura. Isso porque a presença de predadores no ambiente, alimentando-se de insetos-praga suscetíveis ou resistentes, reduz a possibilidade da seleção e desenvolvimento de populações de indivíduos resistentes (DEgRANDE et al., 2002).

$\mathrm{Na}$ cultura do crisântemo ocorrem vários insetos-praga que podem causar injúrias diretas e/ ou indiretas, exigindo, na maioria das vezes, a intervenção do produtor para promover a redução de suas populações a fim de se evitar prejuízos. O uso de inimigos naturais do gênero Orius (Wolff) tem despontado como tática viável para o manejo de pragas nessa cultura, pois são insetos pequenos, com alta capacidade de busca e estão presentes em locais da planta comuns aos de muitas pragas, aumentando as chances de encontro (Bueno, 2000; Argolo et al., 2002; Mendes et al., 2002; Silveira et al., 2003).

Entre as espécies desse gênero, o predador Orius insidiosus (Say) (Hemiptera: Anthocoridae) é um inimigo natural que pode reduzir populações de vários insetos-praga, como tripes, moscas-brancas, afídeos, ácaros, ovos e pequenas lagartas de lepidópteros, podendo ainda alimentar-se de seiva e pólen de plantas (BARBer, 1936; Kiman e Yeargan, 1985; Meiracker e RAMaKers, 1991; Fransen et al., 1993). Em estudo realizado por BuEno et al. (2003), O. insidiosus foi efetivo no controle de tripes em crisântemo, reduzindo em até 35 vezes sua população em relação ao tratamento na ausência do predador. Entretanto, esses autores observaram que a utilização de inseticida à base de deltamethrin para o controle de coleópteros provocou intensa redução da população de $O$. insidiosus liberada, favorecendo o aumento da população de tripes, evidenciando assim a incompatibilidade entre as táticas de controle.

Considerando a importância de predadores do gênero Orius como agentes controladores de pragas em agroecossistemas e em vista da escassez de informações a respeito da seletividade de pesticidas a $O$. insidiosus, o presente trabalho teve por objetivo estudar a toxicidade dos produtos abamectin, acephate, azoxystrobin, benomyl, chlorfenapyr, imibenconazole, iprodione, metalaxyl + mancozeb e triforine para adultos deste predador, em condições de laboratório.

\section{MATERIAL E MÉTODOS}

\subsection{Condução dos bioensaios}

Os espécimes do predador O. insidiosus foram coletados em plantas de picão-preto (Bidens pilosa L.) presentes no Campus da Universidade Federal de Lavras. Os indivíduos coletados foram levados a laboratório, mantidos em câmara climática regulada a $25 \pm 2^{\circ} \mathrm{C}, 70 \pm 10 \%$ de UR e fotofase de 12 horas. Estabeleceu-se uma criação de insetos em laboratório, conforme método descrito por Schmidt et al. (1995) e Bueno (2000). Para a alimentação dos indivíduos foram utilizados ovos da presa alternativa Anagasta kuehniella (Zeller) (Lepidoptera: Pyralidae).

\subsection{Equipamento de pulverização}

A pulverização dos produtos sobre adultos de $O$. insidiosus foi realizada por meio de torre de Potter ajustada à pressão de $15 \mathrm{lb} \mathrm{pol}^{-2}$, assegurando uma aplicação de $1,7 \pm 0,2 \mathrm{mg}$ de calda $\mathrm{cm}^{-2}$. A pulverização foi realizada conforme método recomendado por membros da "International 
Organization for Biological and Integrated Control of Noxious Animals and Plants (IOBC), West Palaearctic Regional Section (WPRS)" (VEIRE et al., 1996; HASSAN, 1997; Degrande et al., 2002).

\subsection{Produtos fitossanitários avaliados}

Os produtos foram utilizados nas concentrações indicadas pelos fabricantes e/ou que estão em fase de pesquisa para o controle de pragas e doenças na cultura do crisântemo. Foram selecionados por serem utilizados para o controle de pragas e doenças em plantios comerciais de crisântemo, em condições de casa de vegetação, na fazenda Terra-Viva (Grupo Schoenmaker), nos municípios de Holambra e Santo Antônio de Posse, Estado de São Paulo.

Os produtos utilizados com suas respectivas dosagens em g i.a $\mathrm{L}^{-1}$ de água foram: abamectin $(0,009)$, acephate $(0,75)$, azoxystrobin $(0,08)$, benomyl $(0,5)$, chlorfenapyr $(1,008)$, imibenconazole $(0,015)$, iprodione $(0,5)$, metalaxyl + mancozeb $(0,028+0,224)$ e triforine $(0,285)$. O tratamento testemunha foi composto apenas por água destilada.

\subsection{Efeito dos produtos sobre adultos de O. insidiosus}

Utilizaram-se 40 adultos (20 machos e 20 fêmeas) com até 24 horas de idade, por tratamento, sendo quatro repetições com dez insetos cada uma, em delineamento inteiramente ao acaso. Esses indivíduos foram obtidos da criação em laboratório e colocados em placas de Petri de $15 \mathrm{~cm}$ de diâmetro e levados à pulverização dos produtos, conforme método descrito no subitem 2.2. Após a pulverização, as placas de Petri contendo os insetos foram fechadas com filme plástico de cloreto de polivinila (PVC) e levados ao laboratório, onde os predadores foram individualizados em placas de Petri de $5 \mathrm{~cm}$ de diâmetro contendo, ad libitum, ovos de A. kuehniella como alimento e algodão umedecido, sendo fechadas com filme plástico de PVC e mantidas em câmara climática nas mesmas condições citadas no subitem 2.1.

A sobrevivência dos adultos foi avaliada 1, 12, 24,48 e 72 horas após a aplicação dos produtos, com o auxílio de um microscópio estereoscópico (40x). Foi considerado morto o adulto que se manteve imóvel ao estímulo gerado pelo toque de um pincel.

Para avaliar o efeito dos produtos na capacidade reprodutiva de adultos tratados, utilizaram-se sete casais de O. insidiosus por tratamento. Para isso, 48 horas após a pulverização dos produtos, os predadores tratados foram separados em casais e cada casal foi colocado em placa de Petri de $5 \mathrm{~cm}$ de diâmetro contendo, ad libitum, ovos de A. kuehniella como alimento e algodão umedecido com água, sendo fechada com filme plástico de PVC.

As placas foram mantidas em câmara climática regulada a $25 \pm 2{ }^{\circ} \mathrm{C}$, UR de $70 \pm 10 \%$ e fotofase de 12 horas. Os casais receberam diariamente uma haste de inflorescência de picãopreto envolvida na base por um chumaço de algodão umedecido em água, para oviposição, por um período de 24 horas e alimento a cada 48 horas. O bioensaio foi realizado em delineamento inteiramente ao acaso, com dez tratamentos e sete repetições, sendo cada unidade experimental composta de um casal de $O$. insidiosus.

A viabilidade dos ovos foi verificada mediante a avaliação das posturas realizadas até o décimo dia após o início do período de oviposição. Após a retirada das hastes das placas realizou-se a contagem do número de ovos colocados e distribuídas em tubos de vidro de $8,5 \mathrm{~cm}$ de comprimento $\times 2,5 \mathrm{~cm}$ de diâmetro, devidamente identificados. No sétimo dia após oviposição, foi realizada a avaliação da viabilidade de cada ovo, sendo considerado viável aquele que tinha o opérculo aberto. Três dias após, os ovos inviáveis foram novamente observados para confirmação ou não de sua inviabilidade.

Foram avaliados os efeitos dos produtos fitossanitários na sobrevivência dos adultos, período de pré-oviposição, número médio diário de ovos e total de ovos colocados em dez dias e viabilidade dos ovos oriundos de fêmeas tratadas.

\subsection{Análises estatísticas}

Os dados referentes à sobrevivência dos indivíduos 1, 12, 24, 48 e 72 horas após a aplicação dos produtos foram transformados para arcoseno $\sqrt{x / 100}$ e submetidos à análise de variância em um modelo de parcelas subdivididas no tempo, com os produtos na parcela. Para os dados balanceados, foi utilizado o proc ANOVA do SAS (SAs Institute, 2001). Para o número diferente de repetições nos tratamentos, utilizou-se o proc GLM do SAS (SAS Institute, 2001). O teste de Tukey a 5\% de significância foi usado para comparar as médias dos tratamentos nos casos em que o teste $F$ foi significativo. 


\subsection{Classificação dos produtos segundo escala de toxicidade estabelecida pela IОВC}

Realizou-se a avaliação da mortalidade total dos indivíduos adultos após o período de contato com os produtos e os dados obtidos foram corrigidos pela fórmula de Аввотт (1925). Em função da mortalidade provocada pelos produtos e seu efeito na capacidade de oviposição das fêmeas tratadas, calculou-se o efeito total (E) utilizando-se da fórmula proposta por VEIRE et al. (1996): $\mathrm{E}=100 \%$ - (100\% - Ma) x ER, em que: $\mathrm{E}$ $=$ efeito total $(\%)$ e $\mathrm{Ma}=$ mortalidade corrigida $\mathrm{em}$ função do tratamento-testemunha (Аввотт, 1925), em que: $\mathrm{Ma}=(\mathrm{Mt}-\mathrm{Mc}) /(100-\mathrm{Mc}) \times 100(\mathrm{Mt}=$ mortalidade observada no tratamento com o produto, $\mathrm{Mc}=$ mortalidade observada no tratamento testemunha), $E R=$ efeito do produto sobre a oviposição: $\mathrm{ER}=\mathrm{Rt} / \mathrm{Rc}(\mathrm{Rt}=$ oviposição média obtida no tratamento com o produto, $\mathrm{Rc}=$ oviposição média obtida no tratamento-controle).

Os produtos foram, então, enquadrados em classes conforme a porcentagem de efeito total observado em : classe 1 = inócuos $(\mathrm{E}<30 \%)$, classe 2 $=$ levemente nocivos $(30 \% \leq \mathrm{E} \leq 80 \%)$, classe $3=$ moderadamente nocivos $(80 \%<\mathrm{E} \leq 99 \%)$ e classe 4 $=$ nocivos $(\mathrm{E}>99 \%)$, conforme escala proposta por membros da IOBC/WPRS (VEIRE et al., 1996; HASSAN, 1997; Degrande et al., 2002).

\section{RESULTADOS E DISCUSSÃO}

\subsection{Efeito dos produtos fitossanitários na sobrevivência de adultos de $O$. insidiosus}

Uma hora após a aplicação, nenhum dos compostos avaliados foi prejudicial aos adultos de $O$. insidiosus (Tabela 1). Doze horas após a pulverização com abamectin, chlorfenapyr, iprodione e metalaxyl + mancozeb observou-se redução significativa na sobrevivência dos indivíduos com médias de $82,5 \%$; $82,5 \% ; 87,5 \%$ e $87,5 \%$ respectivamente. Decorridas 24 horas desde a pulverização, verificou-se sobrevivência menor dos insetos que foram tratados com abamectin $(52,5 \%)$ e acephate $(50 \%)$. Às 48 e 72 horas após a exposição aos compostos constatou-se que abamectin, acephate e chlorfenapyr expressaram maior toxicidade ao predador, com médias de sobrevivência de $25,0 \%$; $17,5 \%$ e $42,5 \%$, e $17,5 \% ; 7,5 \%$ e $40 \%$ respectivamente. Os demais produtos foram inócuos a esta característica biológica.

Resultados semelhantes aos do presente estudo foram observados por VeIRE et al. (1996), que estudaram os efeitos dos resíduos de abamectin e benomyl, pulverizados em placas de vidro, sobre Orius laevigatus (Fieber). Os autores constataram que o primeiro composto foi altamente tóxico para esse percevejo, enquanto o benomyl revelou-se seletivo. Shipp et al. (2000), Morais et al. (2003) e StUdebaKer e KRING (2003), avaliando o efeito de abamectin (1,6 g i.a. ha ${ }^{-1} ; 2,7$ g i.a. ha ${ }^{-1}$ e 10,0 g i.a. ha ${ }^{-1}$ respectivamente) sobre $O$. insidiosus, verificaram que esse composto foi tóxico para o inseto, provocando mortalidade superior a $75 \%$.

O efeito de acephate $\left(1.100 \mathrm{~g}\right.$ i.a. $\left.\mathrm{ha}^{-1}\right)$ sobre O. insidiosus foi avaliado por FUNDERBUK et al. (2000). Constataram que, mesmo em casa de vegetação, o produto foi tóxico para o percevejo. Estudaram ainda o efeito do chlorfenapyr (100 e $200 \mathrm{~g}$ i.a. ha ${ }^{-1}$ ) e observaram que em todas as avaliações realizadas, a densidade de $O$. insidiosus em plantas de pimenta tratadas, para ambas as dosagens, ficou em torno de $36 \%$ daquela encontrada no tratamento-testemunha.

Pulverizando chlorfenapyr (220 g i.a. ha ${ }^{-1}$ ) sobre plantas de algodoeiro para liberação de $O$. insidiosus duas horas após, Pietrantonio e Benedict (1999) verificaram que 48 horas após a exposição do predador às plantas tratadas, ocorreu mortalidade de $55,9 \%$ dos indivíduos. Quando a liberação dos insetos foi realizada 24 horas após o tratamento das plantas, chlorfenapyr também foi prejudicial, causando, após 48 horas de contato, mortalidade de 51,9\%. Em bioensaios de laboratório realizados por ELzEN et al. (1998) foi constatado que resíduos de chlorfenapyr (390 g i.a. ha ${ }^{-1}$ ) presentes em discos foliares de plantas de algodoeiro provocaram redução na população de O. insidiosus. Os autores avaliaram a mortalidade 24 e 72 horas após o contato dos insetos com os resíduos e observaram valores de mortalidade de $50 \%$ e $55,6 \%$ respectivamente.

\subsection{Efeito dos produtos fitossanitários na reprodu-} ção de O. insidiosus

Observou-se aumento no período de préoviposição para os tratamentos com abamectin (5,3 dias) e chlorfenapyr (5,7 dias) em comparação com os demais tratamentos (Tabela 2). O número médio diário de ovos foi reduzido pelo imibenconazole (5,4 ovos), sendo inócuos os demais produtos.

Para fêmeas tratadas na fase adulta não foram observadas diferenças no número médio de ovos em dez dias; em contrapartida, a viabilidade dos ovos foi reduzida nos tratamentos à base de abamectin e acephate, com médias de $65,0 \%$ e $63,6 \%$ de ovos viáveis respectivamente. Azoxystrobin, benomyl, chlorfenapyr, imibenconazole, iprodione, metalaxyl + mancozeb e triforine não afetaram essa característica biológica. 


\subsection{Efeito total dos produtos fitossanitários de acordo com escala proposta pela IOBC}

Os inseticidas abamectin e acephate foram os mais prejudiciais a $\mathrm{O}$. insidiosus, provocando, 72 horas após aplicação, mortalidade de $82,1 \%$ e $89,3 \%$ respectivamente (Tabela 3). Shipp et al. (2000) promoveram o contato de $O$. insidiosus com folhas de pepino tratadas com abamectin e verificaram que até o sexto dia após a aplicação, a mortalidade foi superior a 95\%. Em trabalho realizado por ELzEN et al. (1998), o tratamento de O. insidiosus com profenophos e malathion (produtos do mesmo grupo químico do acephate) causou mortalidade de 51,7\% e $78,9 \%$ respectivamente. Constataram em chlorfenapyr efeito intermediário, promovendo mortalidade de $42,9 \%$ a essa espécie, concordando com os resultados observados neste trabalho.

Tabela 1. Sobrevivência de adultos de Orius insidiosus ( \pm EP) em 1, 12, 24, 48 e 72 horas após a aplicação dos produtos fitossanitários. $\mathrm{T}=25 \pm 2^{\circ} \mathrm{C}, \mathrm{UR}=70 \pm 10 \%$, fotofase $=12$ horas

\begin{tabular}{|c|c|c|c|c|c|}
\hline Produto & $1 \mathrm{~h}$ & $12 \mathrm{~h}$ & $24 \mathrm{~h}$ & $48 \mathrm{~h}$ & $72 \mathrm{~h}$ \\
\hline Abamectin & $97,5 \pm 9,22 a$ & $82,5 \pm 11,22 \mathrm{c}$ & $52,5 \pm 4,08 \mathrm{c}$ & $25,0 \pm 4,16 c$ & $17,5 \pm 2,89 \mathrm{~cd}$ \\
\hline Acephate & $97,5 \pm 9,22 \mathrm{a}$ & $95,0 \pm 8,13 \mathrm{abc}$ & $50,0 \pm 6,45 \mathrm{c}$ & $17,5 \pm 3,18 \mathrm{c}$ & $7,5 \pm 2,22 \mathrm{~d}$ \\
\hline Azoxystrobin & $100,0 \pm 0,00 \mathrm{a}$ & $95,0 \pm 4,92 \mathrm{abc}$ & $75,0 \pm 5,77 \mathrm{bc}$ & $72,5 \pm 5,57 \mathrm{ab}$ & $72,5 \pm 5,57 \mathrm{a}$ \\
\hline Benomyl & $100,0 \pm 0,00 \mathrm{a}$ & $97,5 \pm 3,28 \mathrm{ab}$ & $77,7 \pm 6,45 \mathrm{abc}$ & $72,5 \pm 5,00 \mathrm{ab}$ & $72,5 \pm 5,00 \mathrm{a}$ \\
\hline Chlorfenapyr & $100,0 \pm 0,00 \mathrm{a}$ & $82,5 \pm 6,19 c$ & $72,5 \pm 4,79 \mathrm{bc}$ & $42,5 \pm 6,42 \mathrm{bc}$ & $40,0 \pm 4,77 \mathrm{bc}$ \\
\hline Imibenconazole & $100,0 \pm 0,00 \mathrm{a}$ & $100,0 \pm 0,00 \mathrm{a}$ & $90,0 \pm 5,00 \mathrm{a}$ & $75,0 \pm 4,45 \mathrm{a}$ & $72,5 \pm 6,45 \mathrm{a}$ \\
\hline Iprodione & $100,0 \pm 0,00 \mathrm{a}$ & $87,5 \pm 7,40 \mathrm{bc}$ & $75,0 \pm 6,29 b c$ & $70,0 \pm 6,43 \mathrm{ab}$ & $67,5 \pm 4,07 \mathrm{ab}$ \\
\hline Metalaxyl + mancozeb & $100,0 \pm 0,00 \mathrm{a}$ & $87,5 \pm 6,39 \mathrm{bc}$ & $80,0 \pm 8,16$ abc & $75,0 \pm 7,98$ a & $72,5 \pm 5,84 \mathrm{a}$ \\
\hline Triforine & $100,0 \pm 0,00 \mathrm{a}$ & $90,0 \pm 5,44 a b c$ & $65,0 \pm 4,77 \mathrm{bc}$ & $60,0 \pm 6,11 \mathrm{ab}$ & $57,5 \pm 4,56 \mathrm{ab}$ \\
\hline Testemunha & $100,0 \pm 0,00 \mathrm{a}$ & $100,0 \pm 0,00 \mathrm{a}$ & $85,0 \pm 5,00 \mathrm{ab}$ & $77,5 \pm 6,04 \mathrm{a}$ & $75,0 \pm 6,45 \mathrm{a}$ \\
\hline $\mathrm{CV}(\%)_{\text {parcela }}: 16,14$ & - & - & - & - & CV $(\%)_{\text {subparcela }}: 11,23$ \\
\hline
\end{tabular}

Médias seguidas pela mesma letra na coluna não diferem estatisticamente entre si pelo teste de Tukey $(P>0,05)$.

Tabela 2. Período de pré-oviposição (dias), número médio diário, total de ovos colocados em 10 dias e viabilidade (\%) de ovos $( \pm \mathrm{EP})$ oriundos de fêmeas de Orius insidiosus que entraram em contato com os produtos na fase adulta. Temperatura $=$ $25 \pm 2^{\circ} \mathrm{C}, \mathrm{UR}=70 \pm 10 \%$,fotofase $=12$ horas

\begin{tabular}{|c|c|c|c|c|}
\hline Produto & Pré-oviposição & $\begin{array}{c}\text { Número diário } \\
\text { de ovos }\end{array}$ & $\begin{array}{c}\text { Total de ovos } \\
\text { em } 10 \text { dias }\end{array}$ & Viabilidade \\
\hline & dias & \multicolumn{2}{|c|}{ número } & $\%$ \\
\hline Abamectin & $5,3 \pm 0,25 a$ & $6,4 \pm 0,59 a b$ & $58,3 \pm 6,79 a$ & $65,0 \pm 3,98 b$ \\
\hline Acephate & $4,0 \pm 0,44 b$ & $6,1 \pm 1,03 \mathrm{ab}$ & $55,0 \pm 9,92 \mathrm{a}$ & $63,6 \pm 6,89 b$ \\
\hline Azoxystrobin & $4,0 \pm 0,32 \mathrm{~b}$ & $6,1 \pm 0,50 \mathrm{ab}$ & $59,6 \pm 5,77 \mathrm{a}$ & $75,4 \pm 2,50 \mathrm{ab}$ \\
\hline Benomyl & $4,3 \pm 0,21 \mathrm{~b}$ & $7,0 \pm 0,42 a b$ & $67,6 \pm 4,38 \mathrm{a}$ & $81,1 \pm 2,54 \mathrm{a}$ \\
\hline Chlorfenapyr & $5,7 \pm 0,25 \mathrm{a}$ & $6,7 \pm 0,22 \mathrm{ab}$ & $62,4 \pm 5,00$ a & $76,9 \pm 1,97 \mathrm{ab}$ \\
\hline Imibenconazole & $4,3 \pm 0,21 \mathrm{~b}$ & $5,4 \pm 0,48 b$ & $52,1 \pm 3,00 \mathrm{a}$ & $77,2 \pm 2,18 \mathrm{ab}$ \\
\hline Iprodione & $4,3 \pm 0,21 \mathrm{~b}$ & $6,8 \pm 0,44 a b$ & $67,7 \pm 4,93$ a & $73,3 \pm 2,19 \mathrm{ab}$ \\
\hline Metalaxyl + mancozeb & $4,1 \pm 0,38 b$ & $5,7 \pm 0,53 a b$ & $56,4 \pm 3,57 \mathrm{a}$ & $77,8 \pm 2,41 \mathrm{ab}$ \\
\hline Triforine & $4,1 \pm 0,44 \mathrm{~b}$ & $6,1 \pm 0,56 \mathrm{ab}$ & $59,7 \pm 4,91 \mathrm{a}$ & $75,5 \pm 2,01 \mathrm{ab}$ \\
\hline Testemunha & $4,3 \pm 0,21 b$ & $7,8 \pm 0,37 \mathrm{a}$ & $75,1 \pm 4,78$ a & $79,6 \pm 2,94 \mathrm{a}$ \\
\hline CV (\%) & 10,22 & 15,92 & 18,89 & 9,05 \\
\hline
\end{tabular}

Médias seguidas pela mesma letra na coluna não diferem estatisticamente entre si pelo teste de Tukey $(\mathrm{P}>0,05)$. 
Tabela 3. Mortalidade (\%) corrigida (Ma) de adultos de Orius insidiosus 72 horas após aplicação, efeito total (E\%) e classificação dos produtos pela escala de toxicidade proposta pela IOBC. Temperatura $=25 \pm 2{ }^{\circ} \mathrm{C}, \mathrm{UR}=70 \pm 10 \%$, fotofase $=12$ horas.

\begin{tabular}{|c|c|c|c|c|c|}
\hline Produto & Oviposição $\left({ }^{1}\right)$ & $\mathrm{Rm}\left({ }^{2}\right)$ & $\operatorname{Ma}\left({ }^{3}\right)$ & $\mathrm{E}\left({ }^{4}\right)$ & Classe $\left({ }^{5}\right)$ \\
\hline & - & 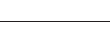 & 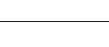 & 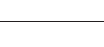 & \\
\hline Abamectin & 22,0 & 11,0 & 82,1 & 81,6 & 3 \\
\hline Acephate & 35,1 & 0,0 & 89,3 & 100,0 & 4 \\
\hline Azoxystrobin & 59,6 & 9,9 & 0,0 & 7,5 & 1 \\
\hline Benomyl & 67,6 & 11,3 & 0,0 & 0,0 & 1 \\
\hline Chlorfenapyr & 59,7 & 11,9 & 42,9 & 36,4 & 2 \\
\hline Imibenconazole & 50,1 & 10,0 & 14,3 & 19,9 & 1 \\
\hline Iprodione & 54,7 & 7,8 & 1,7 & 28,3 & 1 \\
\hline Metalaxyl + mancozeb & 56,4 & 8,1 & 0,0 & 24,9 & 1 \\
\hline Triforine & 58,3 & 11,7 & 25,0 & 18,5 & 1 \\
\hline Testemunha & 75,1 & 10,7 & - & - & - \\
\hline
\end{tabular}

$\left({ }^{1}\right)$ Oviposição média/fêmea em dez dias de avaliação; $\left({ }^{2}\right)$ Número de ovos/fêmea (total de ovos em 10 dias /número de fêmeas sobreviventes); $\left({ }^{3}\right)$ Mortalidade no tratamento corrigida pela fórmula de Аввотт (1925); $\left({ }^{4}\right)$ Efeito total do produto sobre o inimigo natural, onde: $\mathrm{E}=$ $100 \%$ - $\left(100 \%\right.$ - Ma) x ER, onde ER representa o efeito do produto sobre a oviposição; $\left({ }^{5}\right)$ Classe de toxicidade: classe $1=$ inócuo $(E<30 \%) ;$ classe $2=$ levemente nocivo $(30 \% \leq \mathrm{E} \leq 80 \%)$; classe $3=$ moderadamente nocivo $(80 \%<\mathrm{E} \leq 99 \%)$; classe $4=(\mathrm{E}>99 \%)($ VeIRE et al., 1996$)$.

A baixa toxicidade de metalaxyl + mancozeb observada está de acordo com os resultados obtidos por TABORSKY et al. (1995), que observaram o efeito de Ridomil MZ @ sobre Orius majusculus (Reuter) em laboratório e constataram que o produto foi seletivo. Entretanto, LEE et al. (1997) observaram que mancozeb (75 WP) foi altamente tóxico para Orius sauteri Poppius quando aplicado sobre ovos e adultos. As divergências de resultados em relação ao presente trabalho podem ser decorrentes da utilização de espécies diferentes ou, ainda, pelo uso da mistura de metalaxyl + mancozeb e não apenas mancozeb, como no trabalho desenvolvido por LeE et al. (1997).

Em consequência da ação dos produtos fitossanitários sobre a mortalidade e oviposição, calculou-se o efeito total. Verificou-se que acephate afetou drasticamente a sobrevivência de $O$. insidiosus, provocando $100 \%$ de mortalidade. Abamectin foi o segundo composto mais tóxico, registrando-se efeito total de $81,6 \%$. Com base na porcentagem do efeito total, os produtos fitossanitários foram enquadrados em classes, segundo a escala de toxicidade proposta pela IOBC. Os fungicidas azoxystrobin, benomyl, imibenconazole, iprodione, metalaxyl + mancozeb e triforine foram enquadrados na classe 1 (inócuos); chlorfenapyr foi levemente nocivo para $O$. insidiosus (classe 2); abamectin foi moderadamente nocivo (classe 3); acephate, o mais prejudicial, foi enquadrado na classe 4 (nocivo) (Tabela 3 ).

VEIRE et al. (1996) observaram resultados semelhantes para mancozeb e benomyl que, quando aplicados sobres adultos de O. laevigatus, foram enquadrados na classe 1 . Verificaram, ainda, que abamectin foi nocivo para o predador, sendo enquadrado na classe 4 . As divergências de resultados, com relação ao abamectin, comparandose aos do presente estudo, podem estar relacionadas à utilização de espécies diferentes e ao tempo de exposição aos produtos, que foi de 13 dias no trabalho realizado por esses autores.

Foi notável a baixa toxicidade dos fungicidas testados para O. insidiosus. Essa característica assume grande valor no manejo de pragas e doenças na cultura do crisântemo, uma vez que permite o uso desses compostos conjuntamente com o predador.

Os inseticidas testados no presente estudo foram prejudiciais para O. insidiosus, sendo, portanto, recomendada a realização de novos testes em condições de semicampo e campo para a confirmação ou não de sua toxicidade. Nessas condições, a degradação de muitos compostos pode ser acelerada e aliada à menor exposição dos inimigos naturais aos seus resíduos, e contribuir para obtenção da seletividade dos produtos.

\section{CONCLUSÕES}

1. Abamectin, acephate e chlorfenapyr são tóxicos a adultos de $O$. insidiosus, provocando redução na sua sobrevivência.

2. Fêmeas adultas de $O$. insidiosus tratadas com abamectin e acephate têm a viabilidade de seus ovos reduzida, enquanto a aplicação de abamectin e chlorfenapyr prolongam seu período de oviposição. 
3. Os fungicidas azoxystrobin, benomyl, imibenconazole, iprodione, metalaxyl + mancozeb e triforine provocam baixa toxicidade ao $O$. insidiosus.

\section{REFERÊNCIAS}

ABBOTT, W.S. A method of computing the effectiveness of an insecticide. Journal of Economic Entomology, Lanham, v.15, p.265-267, 1925.

ARGOLO, V.M.; BUENO, V.H.P.; SILVEIRA, L.C.P. Influência do fotoperíodo na reprodução e longevidade de Orius insidiosus (Say) (Heteroptera: Anthocoridae). Neotropical Entomology, Londrina, v.31, p.257-261, 2002.

BARBER, G.W. Orius insidiosus (Say), an important natural enemy of the corn earworm". United States Department Agriculture, 1936. (Technical Bulletin, 504).

BUENO, V.H.P. Desenvolvimento e multiplicação de percevejos predadores do gênero Orius Wolff.. In: BUENO, V.H.P. (Ed.). Controle biológico de pragas: produção massal e controle de qualidade. Lavras: UFLA, 2000. p.69-90.

BUENO, V.H.P., van LENTEREN, J.C., SILVEIRA, L.C.P., RODRIGUES, S.M.M. An overview of biological control in greenhouse chrysanthemums in Brazil. IOBC/WPRS Bulletin, Gent, v.26, p.1-5, 2003.

DEGRANDE,P.E.;REIS,P.R.;CARVALHO,G.A.;BELARMINO,L.C. Metodologia para avaliar o impacto de pesticidas sobre inimigos naturais. In:PARRA,J.R.P.; BOTELHO,P.S.M.;CORREA-FERREIRA, B.S. ; BENTO, J.M.S. (Eds.). Controle biológico no Brasil: parasitóides e predadores. São Paulo: Manole, 2002. p.71-94.

ELZEN, G.W.; ELZEN, P.J.; KING, E.G. Laboratory toxicity of insecticide residues to Orius insidiosus, Geocoris punctipes, Hippodamia convergens and Chrysoperla carnea. Southwestern Entomologist., Dallas, v.23, p.335-343, 1998.

FRANSEN, J.J.; BOOGAARD, M.; TOLSMA, J. The minute pirate bug, Orius insidiosus (Say) (Hemiptera: Anthocoridae), as a predator of western flower thrips, Frankliniella occidentalis (Pergande), in chrysanthemum, rose and Saintpaulia. IOBC/ WPRS Bulletin, Gent, v.16, p.73-77, 1993.

FUNDERBURK, J.; STAVISKY, J.; OLSON, S. Predation of Frankliniella occidentalis (Thysanoptera: Thripidae) in field peppers by Orius insidiosus (Hemiptera: Anthocoridae). Environmental Entomology, Lanham, v.29, p.376-382, 2000.

HASSAN, S.A. Métodos padronizados para testes de seletividade com ênfase em Trichogramma. In: PARRA, J.R.P.; ZUCCHI, R. (Ed.). Trichogramma e o controle biológico aplicado. Piracicaba: FEALQ, 1997. p.207-233.

KIMAN, Z.B.; YEARGAN, K.V. Development and reproduction of the predator Orius insidiosus (Hemiptera: Anthocoridae) reared on diets of selected plant material and arthropod prey. Annals of Entomological Society of America, Lanham, v.78, p.464-467, 1985.
LEE, G.H.; CHOI, M.Y.; KIM, D.H. Effect of pesticides on predator Orius sauteri Poppius (Hemiptera: Anthocoridae). Journal of Crop Protection, Suwon, v.39, p.61-66, 1997.

MEIRACKER, R.A.F. van den; RAMAKERS, P.M.J. Biological control of the western flower thrips Frankliniella occidentalis, in sweet pepper, with the anthocorid predator Orius insidiosus. Mededeligen Landbouww Rijksuniv, Gent., v.56, p.241-249, 1991.

MENDES, S.M.; BUENO, V.H.P.; ARGOLO, V.M.; SILVEIRA, L.C.P. Type of prey influences biology and consumption rate of Orius insidiosus (Say) (Hemiptera: Anthocoridae). Revista Brasileira de Entomologia, Londrrina, v.46, p.99-103, 2002.

MORAIS, A.A.; CARVALHO, G.A.; MORAES, J.C.; GODOY, M.S.; COSME, L.V. Avaliação da seletividade de produtos fotossanitários utilizados na cultura do crisântemo a adultos de Orius insidiosus (Say, 1832) (Hemiptera: Anthocoridae) em laboratório. Ciência e Agrotecnologia, Lavras, v.27, p.971-972, 2003.

PIETRANTONIO, P.V.; BENEDICT, J.H. Effect of new chemistry insecticides towards beneficial insects of cotton. In: BELTWIDE COTTON PROD. RES. CONF. NATIONAL COTTON COUNCIL, 1999, Memphis, TN. Proceedings... Memphis, [s. n.], p. 1339-1340.

SAS INSTITUTE. SAS/STAT: users guide. Cary, NC, 2001.

SCHMIDT, J.M.; RICHARDS, P.C.; NADEL, H.; FERGUNSON, $\mathrm{G}$. A rearing method for the production of large numbers of the insidiosus flower bug, Orius insidiosus (Say) (Hemiptera: Anthocoridae). The Canadian Entomologist, Ottawa, v.127, p.445-447, 1995.

SHIPP, J.L.; WANG, K.; FERGUNSON, G. Residual toxicity of avermectin b1 and pyridaben to eight commercially produced beneficial arthropod species used for control of greenhouse pests. Biological Control, San Diego, v.17, p.125131, 2000.

SILVEIRA, L.C.P.; BUENO, V.H.P.; PIERRE, L.S.R.; MENDES, S.M. Plantas cultivadas e invasoras como habitat para predadores do gênero Orius (Wolff) (Heteroptera: Anthocoridae). Bragantia, Campinas, v.62, p.261-265, 2003.

STUDEBAKER, G.E.; KRING, T. Effects of insecticides on Orius insidiosus (Hemiptera: Anthocoridae), measured by field, greenhouse and Petri disc bioassays. Florida Entomologist, Lutz, v.86, p.178-185, 2003.

TABORSKY, V.; ZOHDY, G.I; HEJZLAR, P.; KAZDA, J.; HRUSKA, J. The toxic effect of different pesticides on the predatory bug Orius majusculus (Heteroptera: Anthocoridae). Ochrana-Rostlin, Prague, v.31, p.257-263, 1995.

VEIRE, M. van de; SMAGGHE, G.; DEGHEELE, D. A laboratory test method to evaluate the effect of 31 pesticides on the predatory bug, Orius laevigatus (Heteroptera: Anthocoridae). Entomophaga, Paris, v.41, p.235-243, 1996. 